\title{
Transforming growth factor-1 promotes the transcriptional activation of plasminogen activator inhibitor type 1 in carcinoma-associated fibroblasts
}

\author{
YU ZHU ${ }^{1 *}$, WAN-LE YIN ${ }^{2 *}$, YU-FENG BA ${ }^{3 *}$, LIN TIAN $^{4}$, ZHI-QIANG GU ${ }^{5}$, \\ MING-SHENG ZHANG ${ }^{1}$ and CHU-NAN ZHONG ${ }^{6}$
}

\author{
${ }^{1}$ Orthopedic Department, the Second Affiliated Hospital of Zhengzhou University, Zhengzhou, Henan 450014; \\ ${ }^{2}$ Orthopaedic Trauma and Microsurgery Department, Zhengzhou People's Hospital, Zhengzhou, Henan 450014; \\ ${ }^{3}$ Department of Thoracic Surgery, Affiliated Cancer Hospital of Zhengzhou University (Cancer Hospital of Henan Province), \\ Zhengzhou, Henan 450014; ${ }^{4}$ Department of Internal Medicine, Zhengzhou Ninth People's Hospital, Zhengzhou, \\ Henan 450014; ${ }^{5}$ Department of Nuclear Magnetic Resonance Imaging, the Second Affiliated Hospital \\ of Zhengzhou University, Zhengzhou, Henan 450014; ${ }^{6}$ Orthopedic Department, Henan Provincial People's Hospital, \\ Zhengzhou, Henan 450003, P.R. China
}

Received March 21, 2012; Accepted July 31, 2012

DOI: $10.3892 / \mathrm{mmr} .2012 .1020$

\begin{abstract}
Carcinoma-associated fibroblasts (CAFs) play a pivotal role in promoting the growth, invasion and metastasis of tumor cells. However, to date little is known about the oncogenic mechanisms of CAFs. This study aimed to identify the microenvironmental factors involved in tumor development and progression directed by CAFs in liver metastases. Tissue samples collected from 20 patients with colorectal liver metastases were used in this study. Histological and morphological characterization of the samples was performed using hybridization and immunohistological assays. The mRNA expression of $\alpha$-smooth muscle actin ( $\alpha$-SMA) was measured by northern blotting. The expression of plasminogen activator inhibitor type 1 (PAI-1) was measured by enzyme-linked immunosorbent assay (ELISA). As a result, co-expression of Thy-1 (CD90) and $\alpha$-SMA was identified in CAFs, while normal liver samples were negative for $\alpha$-SMA and Thy-1. Compared with epidermal growth factor (EGF) and tumor
\end{abstract}

Correspondence to: Professor Ming-Sheng Zhang, Orthopedic Department, the Second Affiliated Hospital of Zhengzhou University, 2 Jingba Road, Zhengzhou, Henan 450014, P.R. China

E-mail: zhangms100@126.com

Professor Chu-Nan Zhong, Orthopedic Department, Henan Provincial People's Hospital, 7 Jingwu Road, Zhengzhou, Henan 450003, P.R. China

E-mail: zhongcn2012@126.com

*Contributed equally

Key words: carcinoma-associated fibroblasts, transforming growth factor-1, plasminogen activator inhibitor type 1, tumor, metastasis necrosis factor (TNF) incubation, the expression of $\alpha$-SMA increased significantly following transforming growth factor-1 (TGF-1) incubation $(\mathrm{P}<0.05)$, while platelet-derived growth factor (PDGF) caused a significant suppression of $\alpha$-SMA expression $(\mathrm{P}<0.05)$. PAI-1 expression was significantly lower in unstimulated fibroblasts compared to TGF-1-treated fibroblasts $(\mathrm{P}<0.01)$. The levels of PAI-1 transcription were significantly higher in CAFs from the patient samples compared with the healthy controls. Taken together, our findings suggest that CAFs may be important in migration, matrix degradation, invasion and angiogenesis of tumors, and TGF-1 may promote the activation of PAI-1 transcription in CAFs.

\section{Introduction}

Since 1889 , it has been hypothesized that tumor cells with a special affinity for the surrounding environment were necessary for the growth and spread of cancer (1), and an uneven distribution of breast cancer metastases was observed in different organs and tissues. It was believed that the tumor cells, as 'seeds', would only grow if they were in the matching fabric, the 'soil'. A contemporary definition of the 'seed and soil' hypothesis is based on two principles. Firstly, neoplasms are heterogeneous and composed of cells with different biological properties. Secondly, multiple interactions between tumor cells and host factors cause tumor growth and dissemination (2).

Carcinoma-associated fibroblasts (CAFs) are found in a major proportion of solid tumors and their metastases. These cells, as a heterogeneous group of fibroblasts in many cases, may be activated and have myofibroblastic differentiation (3-5). Certain research has suggested that CAFs play an important pathophysiological role in tumor progression and angiogenesis in vitro and in vivo; thus they are of great significance in the development of targeted treatment strategies (6-10). 
It was believed that CAFs derived from organ-specific local permanent fibroblasts were mediated by cytokines, such as transforming growth factor (TGF)-1 and platelet-derived growth factor (PDGF) $(3,4,11)$. In addition, other potential origins were the transdifferentiation from blood or bone marrow stem cells, and transdifferentiation of mesenchymal epithelial cells was also experimentally described $(12,13)$. In pancreatic tumor tissue of mice, bone marrow was a source of myofibroblasts for $25 \%$ of tumor tissues (14).

Colorectal cancer is one of the main causes of cancer mortality in the world $(15,16)$. The poor prognosis of the disease is caused by the presence of metastases, which occurs mostly in the liver (17). It has been postulated that the microarchitecture and the cellular components of the liver form a favorable environment for the adhesion of tumor cells and the formation of metastases (2). However, little is yet known about the oncogenic mechanisms of CAFs. This study aimed to explore the microenvironmental factors involved in the tumor progression directed by CAFs in liver metastases.

\section{Materials and methods}

Tissue samples and cell culture. The tissue samples were obtained from 20 patients with liver metastases of colorectal cancer. The tissue was obtained from partial hepatic resections. All patients in the study signed an informed consent form. The study protocol was approved by the local research ethics committee of the Second Affiliated Hospital of Zhengzhou University. The tissue samples were mechanically crushed with sterile forceps for $60 \mathrm{~min}$ at $37^{\circ} \mathrm{C}$ in $30 \mathrm{ml}$ of medium 199 (Invitrogen, Carlsbad, CA, USA), and incubated in $1 \mathrm{mg} / \mathrm{ml}$ collagenase type IV (Sigma, Steinheim, Germany). Thereafter, the digested tissue pieces were placed in $30 \mathrm{ml}$ Dulbecco's modified Eagle's medium (DMEM) with $10 \%$ fetal calf serum (FCS, Invitrogen) and $1 \%$ antibiotic/antimycotic solution (Invitrogen), consisting of penicillin, streptomycin and amphotericin B. Subsequently, samples were transferred to $145 \mathrm{~cm}^{2}$ culture plates (Nunclon ${ }^{\mathrm{TM}}$ ) and cultured in a $5 \%$ $\mathrm{CO}_{2}$-containing humidified atmosphere at $37^{\circ} \mathrm{C}$. The first medium change occurred once the cells adhered, after approximately 48 to $72 \mathrm{~h}$.

Immunohistochemical staining. To characterize the tumor-free liver tissue phenotype, the CAFs in human metastatic tissue and tumor-free liver fibroblasts were stained with the following primary antibodies (monoclonal mouse anti-human antibodies) in PBS: $\alpha$-SMA (1/50, clone 1A4, IgG2a; Dako, Glostrup, Denmark), vimentin (1/50, clone V9, Dako), Thy-1 (1/25, clone 5E10, BD Biosciences, Bedford, MA, USA), desmin (1/50, clone D33, Dako), CD45 (1/50, clone T29/33, Dako), cytokeratin 7 (1/50 clone OV-TL 12/30, Dako), cytokeratin 19 (1/100, RCK108 clone, Dako), NCAM (1/25, clone T1, Dako), ICAM-1 (1/50, clone 6.5B5, Dako), laminin (1/25, clone 4C7, Dako) and glial fibrillary acidic protein (GFAP, $1 / 25$, clone $6 \mathrm{~F} 2$, Dako). The staining against CD45 was carried out according to the protocol of DakoCytomation EnVision and System-HRP (Dako Co., Carpinteria, CA, USA).

RNA isolation. In order to extract the total RNA from tissue samples, the RNA Midi kit (Qiagen, Hilden, Germany) was used. The sample was crushed by a disintegrator (MicroDismembrator, Braun Instrumente, Melsungen, Germany), and lysed in $3.8 \mathrm{ml}$ RLT buffer (Qiagen). The lysed sample was centrifuged for $5 \mathrm{~min}$ at 4,000 rpm. Ethanol $(70 \% ; 3.8 \mathrm{ml})$ was added into the supernatant, then the supernatant was planned and mixed for $1 \mathrm{~min}$. To fix the RNA in the membranes of the kit columns, several wash steps were followed according to the manufacturer's instructions. In order to dissolve the RNA from the membrane, the filter was transferred to a new $15 \mathrm{ml}$ collection tube and washed twice with $250 \mathrm{ml}$ of RNase-free water (Sigma). In order to determine the concentration, an aliquot was produced at a dilution of 1/50 in RNase-free water from the kit, and the absorbance was measured at 260 and $280 \mathrm{~nm}$ photometrically (Hach Lange DR 2800 VIS photometer, Germany).

Northern blotting. The transfer of nucleic acids to a nylon membrane was initially from a gel. Based on the concentration gradient of SSC solutions, the transport of RNA was performed by the gel onto the membrane. A total of $20 \mathrm{~h}$ after the transfer was canceled using a UV cross linker $\left(\right.$ Hoefer $^{\mathrm{TM}}$, Amersham Biosciences UK Ltd., UK), the RNA could be linked to the nylon membrane. For subsequent assignment of the bands sizes, the signals of the 18S and 28S rRNA subunits were marked under ultraviolet light. The membrane was either used immediately or sealed and stored in 20X SSC at $4^{\circ} \mathrm{C}$.

Hybridization. Total RNA of the liver samples was transferred to the inward-facing RNA-side of the nylon membrane in hybridization tubes filled with $10 \mathrm{ml}$ EasyHyb hybridization buffer (Roche, USA). The RNA with membrane-covered points was prehybridized for $1 \mathrm{~h}$ at $50^{\circ} \mathrm{C}$ in a rotary hybridization oven (Amersham-Pharmacia, UK). The nonspecific binding of DIG antibody was used to prevent the blocking of the membrane. A hybridization probe was heated for $10 \mathrm{~min}$ at $68^{\circ} \mathrm{C}$ in a water bath, then stored at $-20^{\circ} \mathrm{C}$. After the expiry of prehybridization, buffer was discarded, and the membrane was hybridized at $50^{\circ} \mathrm{C}$ under constant rotation overnight. Following hybridization, the probe was frozen at $-20^{\circ} \mathrm{C}$ again. Thereafter, the membrane was washed twice for $15 \mathrm{~min}$ at $20^{\circ} \mathrm{C}$ in wash buffer $(2 \mathrm{X} \mathrm{SSC}+0.1 \% \mathrm{SDS})$ and twice for $15 \mathrm{~min}$ at $68^{\circ} \mathrm{C}$ in wash buffer $(0.5 \mathrm{X} \mathrm{SSC}+0.1 \% \mathrm{SDS})$.

ELISA. For measurement of PAI-1 concentration in the culture supernatant, the human PAI ELISA kit was used. Firstly, $100 \mu 1$ anti-mouse HRP-conjugated PAI-1 antibodies were placed into each well. The microplate was coated with another monoclonal anti-PAI-1 antibody. The $100 \mu \mathrm{l}$ standard sample was introduced directly into the appropriate wells, after which the immune reaction started. The PAI-1-Ag was bound to a defined epitope on the solid phase-bound monoclonal anti-PAI-1 antibodies and bound to another HRP-conjugated anti-PAI-1 monoclonal antibody defined epitope. Following gentle mixing, the plate was incubated at room temperature for $1 \mathrm{~h}$ and then washed with $300 \mu \mathrm{l}$ wash solution (at least 20 -fold concentrated). After the unbound proteins were washed, $200 \mu 1$ of peroxidase substrate 3,3',5,5'-tetramethylbenzidine (TMB) $/ \mathrm{H}_{2} \mathrm{O}_{2}$ was added for $5 \mathrm{~min}$ at room temperature, and the reaction was stopped with $50 \mu 10.45 \mathrm{M}$ sulfuric acid. Thus the formation of a dye was induced, and the intensity was 
directly proportional to the concentration of the human PAI-1 in the sample. The color stabilization lasted $10 \mathrm{~min}$. The absorbance was read at $450 \mathrm{~nm}$ and the blank value was subtracted. A microplate reader (Dynatech MR 5000) and the software Microwin 3.0 (Microtek) were applied for measurement and evaluation.

Statistical analysis. The result of each experiment was shown as the mean \pm standard deviation (SD) where applicable. Statistically significant differences in each assay were determined by SPSS version 18.0 statistical software package (SPSS Inc., Chicago, IL, USA). Differences in each group were tested for significance using the ANOVA analysis of variance. $\mathrm{P}<0.05$ was considered to indicate a statistically significant difference.

\section{Results}

Morphological and histological characterization of CAFs. Fibroblastic cell populations were classified according to their immunohistochemical marker profile. We observed the cell populations in histological sections of colorectal cancer liver metastases (at least $3 \mathrm{~cm}$ ). The cells present within the metastatic stroma showed differentiation, as occurred in stationary fibroblasts in continuous portal vessels of the liver tissue. Therefore, fibroblasts could be identified by co-expression of vimentin, Thy- 1 and $\alpha$-SMA, while perisinusoidal hepatic stellate cells (HSCs) were localized in the normal liver. Due to the staining in the transition area between the liver tissue and metastases, the phenomenon of fibroblastic cells in the liver tumor could be observed, as shown in Fig. 1A-C, and $\alpha-S M A$, vimentin and Thy-1 were positive in tumor cells.

Clear staining of the marker Thy-1 is shown in Fig. 2. The sections showed marked staining in the septa. Thy-1 positive fibroblasts were distributed throughout the stroma and were intensified in the border region between the tumor and liver tissue (Fig. 2B). In healthy tissue, predominantly the hepatic portal area, but also the vascular wall and bile duct structures were stained (Fig. 2A).

Using a double immunofluorescence stain against $\alpha$-SMA and vimentin, the proportion of $\alpha$-SMA-positive cells was quantified. While all the CAFs were positive for vimentin, the percentage of $\alpha$-SMA-positive cells was between 50 and $90 \%$ (Fig. 3A-B). The majority of the CAFs therefore had a vimentin+/ $\alpha$-SMA+/Thy-1+ fibroblast phenotype. This expression profile matched that of the CAFs in situ. Thus it was concluded that the majority of cultured CAFs was representative of the population found in fibroblastic tumors in situ.

Effect of different growth factors on the expression of $\alpha$-SMA. CAFs are the targets for both cellular growth factors and pro-angiogenic factors. Therefore, CAFs are likely to play a key role in hepatic invasion and metastasis in colorectal carcinoma, as well as endothelial cells. To determine the effect of growth factors on the properties of CAFs, the established cell lines were incubated with increasing concentrations of TGF-1, PDGF, tumor necrosis factor (TNF) and epidermal growth factor (EGF). Subsequently, mRNA was isolated from the cells and analyzed by northern blotting. As shown in Fig. 4A-B, $\alpha$-SMA mRNA expression increased significantly after CAFs
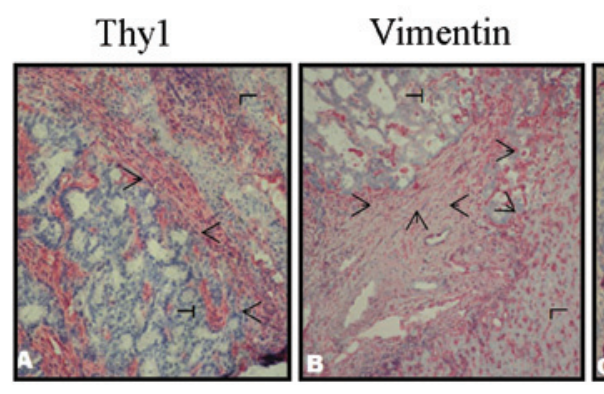

a -SMA

Figure 1. Immunohistochemical labeling of (A) Thy-1, (B) vimentin and (C) $\alpha$-SMA in CAFs in the border region (arrows) between tumor tissue (T) and adjacent hepatic tissue (L) (magnification, x100). $\alpha$-SMA, $\alpha$-smooth muscle actin; CAFs, carcinoma-associated fibroblasts.

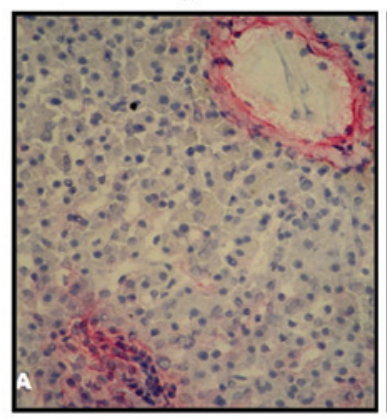

Normal hepatic tissue
Tumor tissue

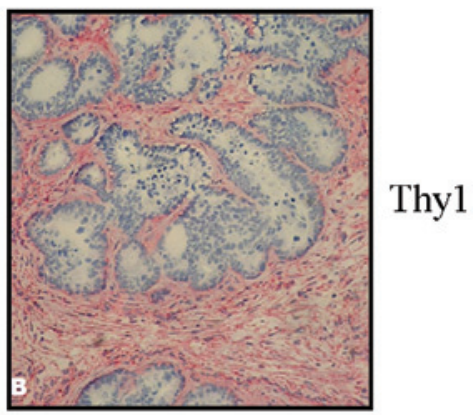

Figure 2. Immunohistological staining of Thy-1 is shown in (A) normal hepatic tissue and (B) tumor tissue (magnification, $\mathrm{x} 400$ ).

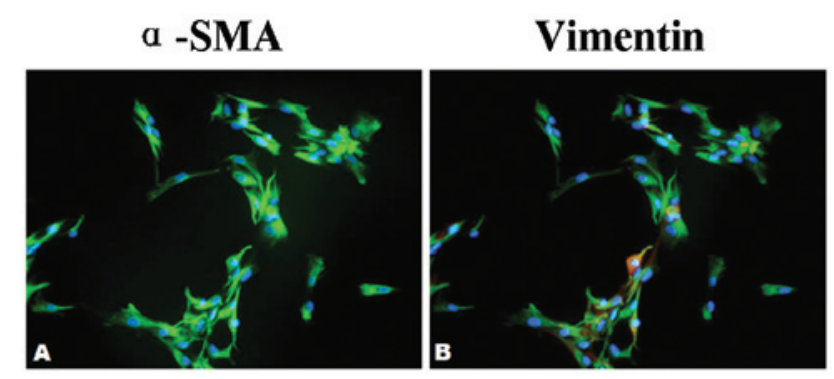

Figure 3. Double-immunofluorescence staining of $\alpha$-SMA and vimentin in CAFs (magnification, x100). $\alpha$-SMA, $\alpha$-smooth muscle actin; CAFs, carcinoma-associated fibroblasts.

were treated with the growth factor TGF-1 $(\mathrm{P}<0.05)$, while EGF and TNF led to no increase in $\alpha$-SMA mRNA expression, and PDGF caused a significant suppression of $\alpha$-SMA expression $(\mathrm{P}<0.05)$.

TGF-1 promotes the expression of PAI-1. Compared with CAFs without stimulation, the PAI-1 mRNA expression significantly increased in those stimulated by TGF-1 $(5 \mathrm{ng} / \mathrm{ml})$, as shown in Fig. 5A and B. The PAI-1 mRNA expression was significantly lower in unstimulated CAFs $(\mathrm{P}<0.01)$.

Secretion of PAI-1. The PAI-1 expression in CAFs from colorectal liver metastases and liver fibroblasts from healthy liver tissue was also analyzed by ELISA, as shown in Fig. 6. 
A
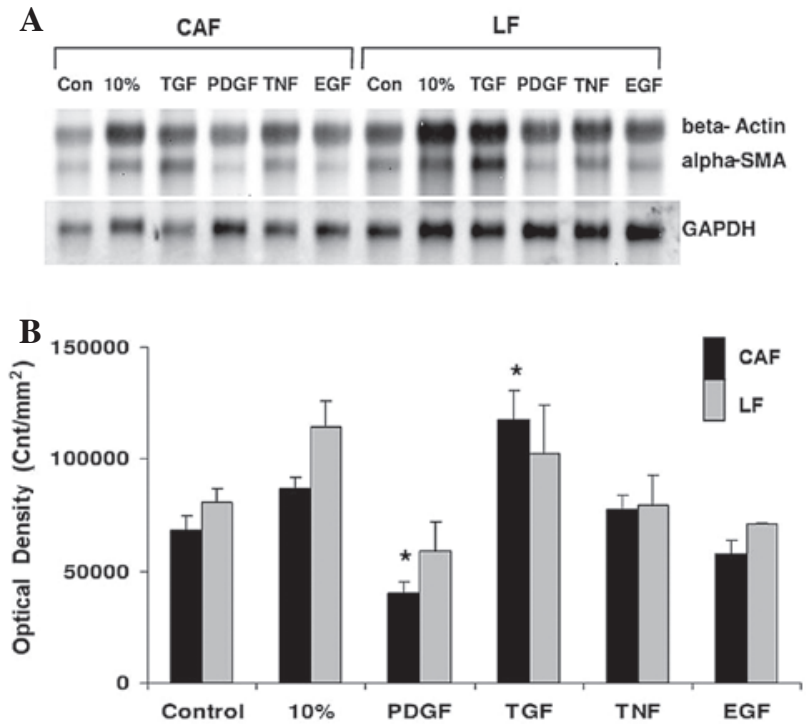

Figure 4. Effect of different growth factors on $\alpha$-SMA expression. (A) $\alpha$-SMA mRNA expression under stimulation by different growth factors. (B) The densitometric analysis of $\alpha$-SMA mRNA expression under stimulation by different growth factors compared with the control ( $\mathrm{P}<0.05)$. CAF, carcinoma-associated fibroblast; LF, liver fibroblasts; $\alpha$-SMA, $\alpha$-smooth muscle actin; PDGF, platelet-derived growth factor; TGF, transforming growth factor; TNF, tumor necrosis factor; EGF, epidermal growth factor.

A

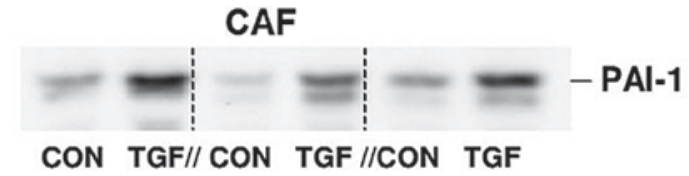

B

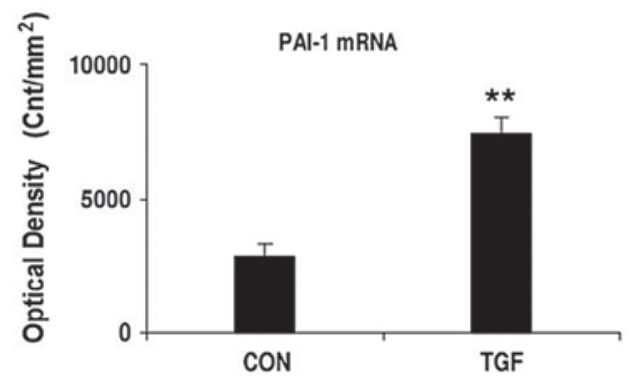

Figure 5. TGF-1 promotes the expression of PAI-1. (A) Influence of TGF-1 on the primary PAI-1 mRNA level. (B) Densitometric analysis of PAI-1 mRNA level in TGF-1-treated CAFs compared with the control $\left({ }^{* * *} \mathrm{P}<0.01\right)$. CAFs, carcinoma-associated fibroblasts; CON, control; PAI-1, plasminogen activator inhibitor type 1; TGF-1, transforming growth factor-1.

The strongest PAI-1 expression was $93.77 \mathrm{ng} / \mathrm{ml}$ in CAFs cultured with $10 \%$ FCS (control, $53.9 \mathrm{ng} / \mathrm{ml}$ ), while it was $79.26 \mathrm{ng} / \mathrm{ml}$ in liver fibroblasts cultured with $10 \% \mathrm{FCS}$ (control, $24.49 \mathrm{ng} / \mathrm{ml}$ ). The influence of different growth factors on cells was strong. The average PAI-1 expression in the fibroblasts from the healthy liver tissues increased to $43.445 \mathrm{ng} / \mathrm{ml}$ under TGF-1 culture with $39.01 \mathrm{ng} / \mathrm{ml}$ for PDGF, while the average PAI-1 expression in CAFs increased to $75.45 \mathrm{ng} / \mathrm{ml}$ under TGF-1 culture, with $59.53 \mathrm{ng} / \mathrm{ml}$ for PDGF. Overall, the PAI-1 production increase in normal tissue was less than that in the metastatic CAFs.

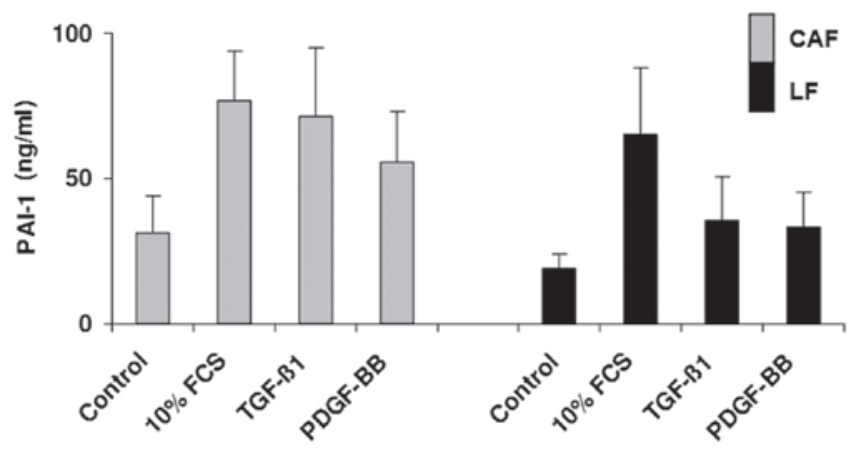

Figure 6. PAI-1 was expressed in tumor and normal tissue, and its increase in production in normal tissue was less than in metastatic CAFs. CAFs, carcinoma-associated fibroblasts; LF, liver fibroblasts; FCS, fetal calf serum; PAI-1, plasminogen activator inhibitor type 1; TGF-1, transforming growth factor-1; PDGF, platelet-derived growth factor.

\section{Discussion}

CAFs are involved in tissue invasion and neo-angiogenesis of tumors in a number of ways. They primarily affect the reorganization of tissue architecture and the production of the tumor stroma (18). They also affect endothelial cell recruitment and vascular infiltration of tumor tissue over pro-angiogenic growth factors (19). In addition, they form the structural basis for tumors (18). Moreover, CAFs are capable of directly affecting the growth of tumor cells (20). Our study demonstrated positivity for vimentin, $\alpha$-SMA and Thy- 1 expression and myofibroblastic differentiation of CAFs as well as the phenomenon of recruitment between liver tumor and healthy liver tissue.

In previous studies, it was assumed that CAFs were transdifferentiated from HSCs (21). Liver fibroblasts could be identified by co-expression of vimentin, Thy- 1 and $\alpha$-SMA, while perisinusoidal HSCs were localized in the normal liver. Our findings suggested that the cultured CAFs and HFs were representative of the respective cell types.

It was clear that the behavior of CAFs of colorectal liver metastases was similar to liver fibroblasts. In fact, it cannot be excluded that the functional similarities of CAFs and liver fibroblasts, and differences between the two cell types decreased continuously during the cultivation. The same change of $\alpha$-SMA expression after growth factor stimulation also showed that CAFs and liver fibroblasts were similar.

More than $95 \%$ of the CAFs and the liver fibroblasts in situ and in vitro were positive for the marker Thy-1, a cell surface glycoprotein, which was found in heterogeneous fibroblast cell isolation from different tissues. The transdifferentiation of fibroblasts into myofibroblasts was attributed to TGF-1 stimulation $(22,23)$. Thy-1 had been considered as a potential differentiation marker between HSCs and portal fibroblasts. However, it was not determined whether activated or transdifferentiated HSCs expressed Thy-1.

The ELISA results showed that PAI-1 expression was very high in CAFs with lower levels in liver fibroblasts. The increases in the metastatic tissue were only slight, possibly reflecting the mixed cell composition. Therefore, CAFs produced a significant proportion of PAI-1. 
Furthermore, the potential role of CAFs was investigated for the invasion of tumor cells, which was not carried out specifically in liver metastases and liver tumors. Following stimulation by growth factors, including PDGF, EGF, TNF and TGF-1, CAFs and liver fibroblasts expressed more PAI-1. The PAI-1 expression was significantly increased by TGF-1. Since the PAI-1 expression in unstimulated cells was much lower, CAFs were stimulated in humans by growth factors (24). Whether these growth factors were secreted by the tumor cells themselves or by other cells involved in tumor growth is currently being studied and pharmacological approaches require exploration (25).

The plasminogen activator (PA) system played a key role in tumor progression, possibly by increasing extracellular matrix degeneration and tumor cell migration. Consequently, urokinase-type plasminogen (u-PA) was discovered as an inhibitor of tumor growth and angiogenesis. Paradoxically, the high concentration of PAI-1 is associated with a poor survival prognosis (26). Through its function as an anti-protease, PAI-1 thus led to proangiogenesis in general to a more aggressive tumor process, and thus to a poorer prognosis (27-29). Furthermore, it was found that tumor cells could be protected from apoptosis by PAI-1 (30).

In conclusion, our findings suggest that CAFs may play an important role in migration, matrix degradation, invasion and thus angiogenesis of tumors. Furthermore, TGF-1 may promote the activation of PAI-1 transcription in CAFs.

\section{References}

1. Welch, DR, Steeg, PS and Rinker-Schaefer CW: Molecular biology of breast cancer metastasis. Genetic regulation of human breast carcinoma metastasis. Breast Cancer Res 2: 408-416, 2000.

2. Duda DG, Duyverman AM, Kohno M, et al: Malignant cells facilitate lung metastasis by bringing their own soil. Proc Natl Acad Sci USA 107: 21677-21682, 2010.

3. Bhowmick NA, Neilson EG and Moses HL: Stromal fibroblasts in cancer initiation and progression. Nature 43: 332-337, 2004.

4. Polanska UM, Acar A and Orimo A: Experimental generation of carcinoma-associated fibroblasts (CAFs) from human mammary fibroblasts. J Vis Exp 56: e3201, 2011.

5. Mazzocca A, Dituri F, Lupo L, et al: Tumor-secreted lysophostatidic acid accelerates hepatocellular carcinoma progression by promoting differentiation of peritumoral fibroblasts in myofibroblasts. Hepatology 54: 920-930, 2011

6. Fujiita M, Hayashi I, Yamashina S, et al: Angiotensin type 1a receptor signaling-dependent induction of vascular endothelial growth factor in stroma is relevant to tumor-associated angiogenesis and tumor growth. Carcinogenesis 26: 271-219, 2005.

7. Fabris VT, Sahores A, Vanzulli SI, et al: Inoculated mammary carcinoma-associated fibroblasts: contribution to hormone independent tumor growth. BMC Cancer 10: 293, 2010.

8. Ishikawa S Takenaka K, Yanagihara K, et al: Matrix metalloproteinase-2 status in stromal fibroblasts, not in tumor cells, is a significant prognostic factor in non-small-cell lung cancer. Clin Cancer Res 10: 6579-6585, 2004.

9. Orimo A, Gupta PB, Sgroi DC, et al: Stromal fibroblasts present in invasive human breast carcinomas promote tumor growth and angiogenesis through elevated SDF-1/CXCL12 secretion. Cell 121: 335-348, 2005.
10. Orimo A and Weinberg RA: Stromal fibroblasts in cancer: a novel tumor-promoting cell type. Cell Cycle 5: 1597-1601, 2006.

11. Rosenthal E, McCrory A, Talbert M, et al: Elevated expression of TGF-betal in head and neck cancer-associated fibroblasts. Mol Carcinog 40: 116-121, 2004.

12. LaRue A, Masuya M and Ebihara Y: Hematopoietic origins of fibroblasts: I. in vivo studies of fibroblasts associated with solid tumors. Exp Hematol 34: 208-218, 2006.

13. Liu C, Chen Z, Chen Z, et al: Multiple tumor types may originate from bone marrow-derived cells. Neoplasia 8: 716-724, 2006

14. Direkze N, Hodivala-Dilke K, Jeffery R, et al: Bone marrow contribution to tumor-associated myofibroblasts and fibroblasts. Cancer Res 64: 8492-8495, 2004.

15. Jemal A, Bray F, Center MM, et al: Global cancer statistics. CA Cancer J Clin 61: 69-90, 2011.

16. Rougier P and Mitry E: Epidemiology, treatment and chemoprevention in colorectal cancer. Ann Oncol 14: ii3-5, 2003.

17. Tajima Y, Ishibashi K, Ishiguro T, et al: Analysis of hepatic lymph node metastasis in liver metastases from colorectal cancer. Gan To Kagaku Ryoho 38: 2228-2231, 2011 (In Japanese).

18. Olaso E, Salado C, Egilegor E, et al: Proangiogenic role of tumor-activated hepatic stellate cells in experimental melanoma metastasis. Hepatology 37: 674-685, 2003.

19. Claffey KP, Abrams K, Shih SC, et al: Fibroblasts growth factor 2 activation of stromal cell vascular endothelial growth factor expression and angiogenesis. Lab Invest 81: 61-75, 2001.

20. Lee HO, Mullins SR, Franco-Barraza J, et al: FAP-overexpressing fibroblasts produce an extracellular matrix that enhances invasive velocity and directionality of pancreatic cancer cells. BMC Cancer 11: 245, 2011.

21. Gulubova MV: Ito cell morphology, alpha-smooth muscle actin and collagen type IV expression in the liver of patients with gastric and colorectal tumors. Histochem J 32: 151-164, 2000.

22. Koumas L, Smith TJ, Feldon S, et al: Thy-1 expression in human fibroblast subsets defines myofibroblastic or lipofibroblastic phenotypes. Am J Pathol 163: 1291-1300, 2003.

23. Barker TH, Grenett HE, MacEwen MW, et al: Thy-1 regulates fibroblast focal adhesions, cytoskeletal organization and migration through modulation of p190 RhoGAP and Rho GTPase activity. Exp Cell Res 295: 4884-4896, 2004.

24. Wakahara K, Kobayashi H, Yagyu T, et al: Transforming growth factor-beta1- dependent activation of $\mathrm{Smad} 2 / 3$ and up-regulation of PAI-1 expression is negatively regulated by Src in SKOV-3 human ovarian cancer cells. J Cell Biochem 93: 437-453, 2004.

25. Hirashima Y, Kobayashi H, Suzuki M, et al: Transforming growth factor-betal produced by ovarian cancer cell line HRA stimulates attachement and invasion through an up-regulation of plasminogen activator inhibitor type-1 in human peritoneal mesothelial cells. J Biol Chem 278: 26793-26802, 2003.

26. Halamkova J, Kiss I, Pavlovsky Z, et al: Clinical significance of the plasminogen activator system in relation to grade of tumor and treatment response in colorectal carcinoma patients. Neoplasma 58: 377-385, 2011.

27. Zubac DP, Wentzel-Larsen T, Seidal T, et al: Type 1 plasminogen activator inhibitor (PAI-1) in clear cell renal cell carcinoma (CCRCC) and its impact on angiogenesis, progression and patient survival after radical nephrectomy. BMC Urol 10: 20, 2010.

28. Grebenchtchikov N, Maguire TM, Riisbro R, et al: Measurement of plasminogen activator system components in plasma and tumor tissue extracts obtained from patients with breast cancer: an EORTC Receptor and Biomarker Group. Oncol Rep 14: 235-239, 2005

29. Nishioka N, Matsuoka T, Yashiro M, et al: Linoleic acid enhances angiogenesis through suppression of angiostatin induced by plasminogen activator inhibitor 1. Br J Cancer 105: 1750-1758, 2011.

30. Romer MU, Due AK, Larsen JK, et al: Indication of a role of plasminogen activator inhibitor type I in protecting murine fibrosarcoma cells against apoptosis. Thromb haemost 94: 859-866, 2005. 